\title{
Integrated Metabolomic and Transcriptomic Analysis of the Anthocyanin and Proanthocyanin Regulatory Networks in Red Walnut Natural Hybrid Progeny Leaves
}

\author{
Lei Wang \\ Henan Agricultural University \\ Lin Li \\ Henan Agricultural University \\ Wei Zhao \\ Henan Agricultural University \\ Haijun Meng \\ Henan Agricultural University \\ Ganggang Zhang \\ Henan Agricultural University \\ Wenjiang Wu \\ Henan Agricultural University \\ Jiangli Shi \\ Henan Agricultural University \\ Guoliang Wu ( $\nabla$ walnut-wu@126.com ) \\ Henan Agricultural University
}

\section{Research Article}

Keywords: transcriptome, metabolome, anthocyanin, proanthocyanin, red walnut

Posted Date: December 28th, 2021

DOI: https://doi.org/10.21203/rs.3.rs-1089011/v1

License: (c) (i) This work is licensed under a Creative Commons Attribution 4.0 International License. Read Full License 


\section{Abstract}

\section{Background}

Walnuts are one of the most important dry fruit crops worldwide, typically exhibiting green leaves and yellow-brown or gray-yellow seed coats. A specific walnut type, red walnut 'RW-1', with red leaves and seed coats was selected as the plant material because of its higher anthocyanin and proanthocyanin (PA) contents. Anthocyanins and PAs coprise important secondary defense methods for plants to respond to biotic and abiotic stresses. However, few studies have focused on the molecular mechanism of anthocyanin biosynthesis in walnuts.

Results

From the results of widely targeted metabolome and anthocyanidin detection analysis, 395 substances, including 4 PAs and 26 anthocyanins, were identified from the red-leaf walnuts of RW- 1 natural hybrid progenies (SR) and the green-leaf walnuts of RW-1 natural hybrid progenies (SG). Among these, all anthocyanin types in SR were significantly upregulated compared with SG. Additionally, delphinidin 3-Ogalactoside, cyanidin 3-O-galactoside, delphinidin 3-O-glucoside and cyanidin 3-O-glucoside were identified as the primary components of anthocyanidins because of their higher contents. Nine anthocyanidins, malvidin 3-O-galactoside, malvidin 3-O-arabinoside, cyanidin 3-O-(6-O-malonyl-beta-Dglucoside), delphinidin 3-O-glucoside, delphinidin 3,5-O-diglucoside (Delphin), peonidin 3-O-(6-O-malonylbeta-D-glucoside), petunidin 3-O-(6-O-malonyl-beta-D-glucoside), petunidin 3-O-arabinoside and pelargonidin 3-O-(6-O-malonyl-beta-D-glucoside), were detected only in the SR walnuts. For PAs, proanthocyanin $\mathrm{C} 1$ was upregulated in SR compared with SG, while proanthocyanin B1 and proanthocyanin B3 were upregulated in SR-1 and SR-3 but downregulated in SR-2 compared with the controls. Furthermore, transcriptome analysis demonstrated that the expression of structural genes $(\mathrm{C} 4 \mathrm{H}$, $F 3 H, F 3^{\prime} 5^{\prime} H, U F G T S, L A R$ and $\left.A N R\right)$, four MYBs and six WD4Os in the anthocyanin and PA biosynthetic pathways were significantly higher in the SR walnut.

\section{Conclusions}

Our results provide valuable information on anthocyanin and PA metabolites and candidate genes in anthocyanin and PA biosynthesis, which provides new insights into anthocyanin and PA biosynthesis in walnuts.

\section{Background}

Anthocyanins and proanthocyanins (PAs) are important secondary metabolites. At present, more than 635 kinds of anthocyanins and 680 kinds of proanthocyanins have been identified [1]. Anthocyanin is one of the most important color-presenting materials in flavonoids, mainly existing in the leaves, flowers, and fruits of higher plants, and is formed by the combination of anthocyanidin and glycosyls [2]. Cyanidin (Cy), delphinidin (Dp), pelargonidin (Pg), peonidin (Pn), petunidin (Pt), and malvidin (Mv) are six 
common anthocyans, and common glycosyl includes glucose, galactose, sucrose, etc. [3]. Various types and amounts of glycosyls bound to separate positions of anthocyanidin resulted in a significant increase in anthocyanin compounds [4]. PAs are oligomers or polymers polymerized by flavane-3-alcohol subunits, and their degree of polymerization may vary according to plant species [5]. The structural changes of PAs depend on the properties of the flavan-3-alcohol initiator and extension unit, the position and stereo configuration of the link with the lower unit, the degree of polymerization, and whether there is modification. These factors increase the structural diversity of PAs [6]. Anthocyanins and PAs act to protect plants from ultraviolet ray damage, attract insect pollination, resist low temperatures $[7,8]$, and contribute to colors [9].

The anthocyanin and PA biosynthesis pathways have been well characterized in some plants [10]. Generally, phenylalanine is regarded as the initial biosynthesis of these two substances, which is composed of a series of enzymatic reactions, including phenylalanine ammonia lyase (PAL), cinnamate 4-hydroxylase $(\mathrm{C} 4 \mathrm{H})$, chalcone synthetase $(\mathrm{CHS})$, chalcone isoenzyme $(\mathrm{CHI})$, flavanone 3-hydroxylase $(\mathrm{F} 3 \mathrm{H})$, flavonoid 3',5'-hydroxylase $\left(\mathrm{F}^{\prime} \mathrm{5}^{\prime} \mathrm{H}\right)$, and dihydroflavonol 4-reductase (DFR). Anthocyanin and PA are separated, the anthocyanin is biosynthesized by anthocyanin synthetase (ANS) and UDP glucose: flavonoid-3-0-glucosyltransferase (UFGT) [11, 12], while PAs are biosynthesized by leucoanthocyanidin reductase (LAR) and anthocyanidin reductase (ANR) [13].

Meanwhile, the structural genes in anthocyanin and PA biosynthesis are regulated by multiple transcription factors, among which MYB transcription factors have been widely studied. The grape R2R3MYB TFs VvMYBA1, VvMYBA6 and VvMYBA7 regulate anthocyanin biosynthesis by activating UFGT and $3 A T$ expression [14]. The overexpression of $M d M Y B 3$ increases anthocyanin accumulation in tobacco by activating the gene expression of CHS, CHI, UFGT and FLS[15]. The overexpression of GhMYB1a in gerbera and tobacco (Nicotiana tabacum) decreases anthocyanin accumulation by upregulating the structural gene expression of NtCHS, NtF3H, NtDFR, NtANS, and NtUFGT [16]. For PA biosynthesis, AtTT2, a positive R2R3 MYB regulator in Arabidopsis, followed the same expression pattern as AtANR and influenced the PAs in seed coats [17]. Meanwhile, there have been a large number of reports on AtTT2 homologous genes in other species, including $V v M y b P A 1$ and $V v M y b P A 2$ in Vitis vinefera $[18,19]$, FtMYB1 and FtMYB2 in Fagopyrum tataricum [20], MtMYB14 in Medicago truncatula [21], and GhMYB10 and GhMYB36 in Gossypium hirsutum [22].

MYB regulates anthocyanin and PA biosynthesis either alone or by forming the MBW complex (MYBbHLH-WD40) [23, 24]. For example, AcMYB123 and AcbHLH42 in Actinidia chinensis cv. Hongyang promotes anthocyanin accumulation by activating the promoters of ACANS and Ac3FGT1 [25]. PyMYB10 and PyMYB114 cotransformed with PybHLH3 induced the promoter activity of PyDFR, PyANS, PyUFGT, PyGST and PyABC transporters [26]. Composed of AtMYB123 (TT2), AtbHLH42 (TT8), and TTG1 proteins in Arabidopsis, it was reported to activate the expression of DFR, LDOX, and ANR genes, which led to the accumulation of PAs in the seed coat [27]. FaMYB9/11, FabHLH3, and FaTTG1 in strawberry [28] and DkMYB2/4, DkMYC1, and DkWDR1 in persimmon [29] are transcriptional activation complexes of PA biosynthesis pathways, which indicates that MBW complexes are well conserved in plants [30]. Therefore, 
the present study aimed to elucidate the regulatory mechanism of the main structural genes and transcription factors involved in anthocyanin accumulation based on red and green walnuts.

Walnuts (Juglans regia L.) are produced by an ancient fruit tree from the Juglandaceaefamily. They rank in the top four nuts in the world, and the walnut tree is considered to be an important ecological tree. The walnut industry has been included as the core point of industrial poverty alleviation in many areas and plays an important role in helping to target poverty alleviation and to increase farmers' income. At present, the phenotypic traits of walnut varieties are similar, with green leaves and yellow-brown or grayyellow seed coats. Luckily, a red walnut accession 'RW-1' was found by our research group with a red colored leaf, pericarp, seed coat and xylem owing to high anthocyanin content [31]. In our previous studies, we found that the growth of red walnut was less than that of ordinary walnut, which was caused by weak photosynthesis [32]. The enrichment of anthocyanins in red walnut can not only improve fruit quality but also weaken the growth potential of trees and reduce the need for pruning, which is in line with the development trend of dwarf and dense planting fruit trees. Although several genes, such as $b H L H s$ and $C H S S$, have been identified in anthocyanin biosynthesis [33, 34], the molecular mechanism of anthocyanin and PA biosynthesis has not yet been clearly elucidated in red walnut.

In recent years, the combination analysis of the transcriptome and metabolome has been widely used to clarify anthocyanin and PA biosynthesis and accumulation in plants [13,35-40]. In the current study, the regulatory networks of anthocyanin and PA biosynthesis in walnut were constructed using two 'RW-1' natural hybrid accessions with red and green leaves, respectively. The comparative analysis of metabolomics and transcriptomics aimed to elucidate the pathway of anthocyanin and PA metabolites and to identify the differentially expressed genes (DEGs) in walnut anthocyanin and PA biosynthesis.

\section{Results}

\section{Widely targeted metabolome and anthocyanidin detection in red walnut natural hybrid progeny leaves over developmental stages}

To maintain consistency of the genetic background, the RW-1 natural hybrid progenies SG and SR walnuts with separate leaf colors were investigated. During the young leaf period, the SR walnut leaves were completely red, while the SG walnut leaves were green. Next, the color difference between SR leaves and SG leaves became inconspicuous with leaf development. The SR leaves retained the red color only in the leaf vein; in contrast, the SG leaves only showed green color at all times (Fig. 1a).

The metabolome of 18 samples was profiled by a Widely Targeted Metabolome and anthocyanidin detection approach. A total of 395 compounds in the walnut leaves were classified into 19 classes (Table S2), including 26 anthocyanins and 4 proanthocyanins. The quality of the Widely Targeted metabolome data was reliable, as evidenced by the PCA results (Fig. S2a, b) and the correlation analysis of population samples (Fig. S3) of the time course and two comparative analyses. 
As Fig. 1b shows, 88, 20 and 36 DAMs among SG-1 vs. SR-1, SG-2 vs. SR-2, and SG-3 vs. SR-3 were identified between paired samples, respectively. Furthermore, the histogram showed that 30 upregulated metabolites, 58 downregulated in the first stage, 13 upregulated while 7 downregulated in the second stage, and 22 upregulated while 14 downregulated in the third stage (Fig. 1c). The enrichment analysis of KEGG pathways showed that the most significant enrichment pathways in all three stages were anthocyanin biosynthesis (Fig. S4).

\section{Components and contents of flavonoids and anthocyanins during walnut leaf development stages}

In this work, a total of 88 flavonoid metabolites were identified, including 55 flavonoids, 3 isoflavonoids, 26 anthocyanins and 4 proanthocyanins.

To determine whether the red pigmentation of red walnut is caused by anthocyanins, we analyzed the soluble anthocyanins in green and red leaves using a UPLC-ESI-MS/MS system. A total of 26 anthocyanins were detected in walnut leaves and were significantly upregulated in SR-1 compared with SG-1; meanwhile, only 11, 13 and 12 anthocyanins were detected in SR-1, SR-2 and SR-3 compared with the controls, respectively (Fig. 1d). Furthermore, delphinidin 3-O-galactoside, cyanidin 3-O-galactoside, delphinidin 3-O-glucoside and cyanidin 3-O-glucoside were the primary components of anthocyanidins because of their higher contents. We also found that 9 anthocyanin compounds only existed in red leaves, including malvidin 3-O-galactoside, malvidin 3-O-arabinoside, cyanidin 3-O-(6-O-malonyl-beta-Dglucoside), delphinidin 3-O-glucoside, delphinidin 3,5-O-diglucoside (Delphin), peonidin 3-O-(6-O-malonylbeta-D-glucoside), petunidin 3-O-(6-O-malonyl-beta-D-glucoside), petunidin 3-O-arabinoside and pelargonidin 3-O-(6-O-malonyl-beta-D-glucoside).

PAs, including proanthocyanin B1, proanthocyanin B2, proanthocyanin B3 and proanthocyanin C1, were detected in the walnut leaves. proanthocyanin C1 was upregulated in SR compared with SG, while proanthocyanin B1 and proanthocyanin B3 were upregulated in SR-1 and SR-3 but downregulated in SR-2 compared with the controls (Fig. 1d). Moreover, 8 downaccumulated flavonoids contents were found to be significantly different in SG-1 vs. SR-1, 2 downaccumulated and 1 upaccumulated in second period, while 2 downaccumulated and 3 upaccumulated in third period (Fig. S5). In summary, these patterns of pigment accumulation were consistent with the strikingly different leaf color phenotypes of SR and SG.

\section{Differentially expressed genes between green- and red-leaf walnuts}

RNA sequencing (RNA-Seq) was used to profile genome-wide gene expression and transcriptome changes during leaf development. With three biological replicates, transcriptome sequencing of the 18 walnut leaf samples yielded a total of $134.01 \mathrm{~Gb}$ clean data with $95.22 \%$ of bases scoring Q30. Furthermore, $92.23-95.27 \%$ of the total clean reads were unique matches with the walnut reference genome, and 4,131 novel genes were identified, including 3,154 annotated genes [33]. 
From Fig. 2a, 5,708, 1,587 and 703 DEGs were obtained from SG-1 vs. SR-1, SG-2 vs. SR-2, and SG-3 vs. SR-3, respectively, containing 24 identical genes (Fig. 2a). According to the results of transcriptomic analysis, there were 5,708 DEGs between SG-1 and SR-1, of which 3,513 were upregulated and 2,195 were downregulated. There were 1,587 DEGs between SG-2 and SR-2, including 810 upregulated genes and 777 downregulated genes. There were 703 DEGs between SG-3 vs. SR-3, including 364 upregulated genes and 339 downregulated genes. The profiles of the DEGs indicated that the differences in gene expression affected leaf color in walnuts. To better understand biological functions of DEGs in different color leaves, GO analysis were applied (Fig. 2b-d). The DEGs were enriched in biological process, cellular component and molecular function according to GO database. In the biological process category, most of DEGs were annotated to metabolic process, cellular process and single-organism. With respect to the cellular component category, DEGs were mainly assigned to cell, cell part and membrane. In the molecular function category, DEGs were primarily enriched in catalytic activity, binding and transporter activity.

\section{Analysis of structural genes involved in anthocyanin and PA biosynthesis.}

On the basis of a reported anthocyanin biosynthesis pathway, a pathway diagram that included the expression heat map of each structural gene in the anthocyanin biosynthesis pathway of walnut were constructed (Fig. 3). In view of leaf color differences in appearance at Stage 1, the combination analysis of transcriptomic and metabolomic data explained the anthocyanin biosynthesis pathway in SG and SR walnuts. The results showed that 17 DEGs were involved in the anthocyanin biosynthesis pathway, and 4 DEGs were involved in the PA biosynthesis pathway. Structural genes, including $\mathrm{C} 4 \mathrm{H}$ genes (gene40343 and gene42522), CHS genes (gene4994, gene39336, gene35863 and gene32601) [34], F3Hgene (gene40994), F3'5'H gene (gene4387), ANS gene (gene1297) and UFGT genes (gene28510, gene35146, gene1870, gene24302, gene35144, gene1697, and gene36923), were all upregulated, and only one UFGT gene (gene35048) was downregulated in red leaves (Fig. 3). For the biosynthetic pathway of PA, LAR and ANS, ANR and UFGT compete for substrates, and two LAR genes (gene38150, gene640) and one ANR gene (gene24378) were upregulated, while one ANR gene (gene21099) was downregulated in red leaves.

\section{Identification of TFs Related to Anthocyanin and PA Biosynthesis}

Anthocyanin and PA biosynthesis are regulated by the MBW (MYB-bHLH-WD40) complex. As shown in Fig. 4a, MYB and bHLH ranked as the top two TFs related to anthocyanin biosynthesis in walnut leaves. Based on the Arabidopsis MYB protein domains, 135 putative walnut MYB protein sequences were obtained with default parameters using HMMER and BLASTP (Table S3). A phylogenetic tree was constructed using the $135 \mathrm{JrMYBs}$ and MYBs of other species related to anthocyanin and PA biosynthesis (Fig. 4b). Nine subfamilies and $25 \mathrm{JrMYBs}$ were obtained from the current study. Based on a false discovery rate (FDR) 0.05 and $\left|\log _{2} \mathrm{FC}\right| \geq 1.5,3$ differentially expressed $M Y B$ genes related to anthocyanin biosynthesis in red frames, JrMYB1b (gene38312), JrMYB6a (gene32351), JrMYB123 (gene9445), and one MYB gene related to PA biosynthesis, JrTT2 (gene39085), were screened (Fig. 4c). The bHLHs involved in anthocyanin biosynthesis have been reported by our research group, including 
JrbHLHA1, JrbHLHA2, JrEGL 1a, and JrEGL 1b [33]. For WD40, we obtained 23 DEGs, including 6 upregulated (gene1567, gene24750, gene31286, gene32032, gene42048, and gene5786) and 10 downregulated genes (gene10090, gene1113, gene13751, gene15328, gene16177, gene16178, gene1631, gene3696, gene37190, and gene38133) in the first stage, 8 upregulated (gene15790, gene22436, gene37190, gene37530, gene39719, gene5160, gene7486, and gene9207) and 1 downregulated gene (gene3696) in the second stage, and 1 downregulated gene (gene3696) in the third stage. Among them, gene3696 showed a downregulated trend in all three stages. These TFs may exert an effect on or participate in the regulation of structural and regulatory genes in anthocyanin biosynthesis.

\section{qPCR analysis of DEGs related to anthocyanin and PA biosynthesis}

Based on our above results, 12 differentially expressed structural genes involved in the anthocyanin biosynthetic pathway and $4 M Y B$ genes were analyzed using qPCR methods. The results in Fig. S6 show that eight structural genes, JrC4Hs (gene40343, gene42522), JrF3'5'H (gene4387), JrUFGTs (gene35144, gene35146, gene1697, gene24302 and gene1870), and four MYB genes (gene38312, gene32351, gene9445, gene39085), were highly expressed in red leaf walnut at the first stage, which indicated that the high expression of genes involved in anthocyanin biosynthesis was related to the color of red walnut leaves. It was also further suggested that the transcriptome data were accurate and consistent with the expression of genes related to anthocyanin biosynthesis in red walnut.

\section{Discussion}

The primary pigments in red walnut have been identified as flavonoids, particularly anthocyanins [31, 34], and previous studies on walnut coloration are limited. The current work aimed to explore more comprehensive metabolites involved in the color changes in walnut leaves, and 395 metabolites were obtained from walnut leaves using the widely targeted metabolomics and anthocyanidin detection approach (Table S2). This was the first study to present a genome-wide examination of anthocyanins and the gene expression profiles of walnuts, aiming to provide a more comprehensive landscape of the metabolites involved in the color changes in walnut leaves during development. Moreover, we also found that PA accumulation was higher in SR than in SG, especially in the first stage (Fig. 1d). By transcriptomic and metabolic analysis, 21 core genes were identified in anthocyanin and PA biosynthesis (Fig. 3). These findings provide a theoretical basis for further study of the mechanism of red walnut.

Anthocyanins are secondary metabolites in plants, such as ornamental plants, fruits, vegetables and medical plants, and play various roles in many biological processes, including determining fruit quality and flower colors, improving resistance, and avoiding UV and strong light damage [41, 42]. In addition to the rich nutrition in kernels, walnuts are also an important ecological tree. Red walnuts RW-1 possesses great ornamental value, nutritional value and economic benefits. A total of 26 anthocyanins were detected in green- and red-leaf walnuts (Fig. 2), and cyanidin 3-0-galactoside, delphinidin 3-0-galactoside, and delphinidin 3-0-glucoside were the main anthocyanins in SR. Importantly, the anthocyanin content 
was consistent with leaf color. Interestingly, 6 anthocyanin compounds, cyanidin, delphinidin, malvidin, pelargonidin, peonidin, and petunidin, existed not only in SR but also in SG (Fig. 2). Similar results were also found in white Salvia miltiorrhiza flowers [43]. The color depended on the types and contents of anthocyanins, copigment, chlorophyll, vacuole pH, and metal ions [44]. The 6 anthocyanins accumulated less in green leaf walnuts than in red leaf walnuts, suggesting that complete anthocyanin metabolic pathways were also present in SG. The other factors affecting the color of walnut leaves need to be studied further.

The core genes for walnut anthocyanin and PA biosynthesis involve multiple enzymes encoded by structural genes (C4H, CHS, CHI, F3H, F3' H, F3'5'H, DFR, ANS, UFGT, LAR and ANR) [44]. The results from this study showed that the expression levels of $C 4 H s, C H S s, F 3 H$ and $F 3^{\prime} 5^{\prime} H$ in SR were significantly higher than those in SG (Fig. 3, Fig. S6), which are common to both anthocyanin and PA biosynthesis pathways. The expression of ANS and UFGT genes in SR was significantly higher than that in SG (Fig. 3, Fig. S6), suggesting that the genes mainly regulated red coloration in red leaf walnuts. As walnut leaves develop, these structural genes are downregulated, and the color tends towards green. The UFGT gene is involved in the final steps of the flavonoid biosynthetic pathway (biosynthesis and accumulation of anthocyanins). The expression of UFGT genes (gene35144, gene35146, gene1697, gene24302 and gene1870) was higher in SR than in SG at Stage 1, and UFGT gene (gene35038) was the opposite. It is worth noting that the 3 UFGT gene trends during the 3 stages corresponded to the color change in walnut leaves. Anthocyanidins are extremely unstable and easy to degrade; therefore, glycosylation is important to stabilize. As the last step of the anthocyanin biosynthesis pathway, UFGTs are considered to be the key enzymes controlling anthocyanin biosynthesis in many plants, and they play an important role in anthocyanin metabolism [11, 45-47]. The present study first explored the expression profile of UFGTS using red walnuts, providing new insights for studying UFGT gene function. The expression of $L A R$ and $A N R$ was also higher in SR than in SG, suggesting that these genes may play important roles in PA biosynthesis (Fig. 3, Fig. S6).

Anthocyanin biosynthesis is regulated by the MBW (MYB-bHLH-WD40) complex. In the MBW complex, bHLH and MYB transcription factors have DNA binding functions and can specifically bind to the promoters of structural genes in the anthocyanin biosynthesis pathway, while the WD40 protein plays a stable role in the MBW complex $[23,48]$. Actinidia chinensis Planch AcMYB123 and AcbHLH42 promote anthocyanin accumulation in the inner pericarp of Actinidia chinensis Planch by activating the promoters of ACANS and ACF3GT1 [25]; PyWRKY26 and PybHLH3 act together on the PyMYB114 promoter, and cotransfection can activate the expression of PyUFGT to regulate the accumulation and transportation of anthocyanin in pear [26]. In this study, we identified five MYBs, four bHLHs and six WD40s based on their expression levels and phylogenetic analysis. The manner in which TFs play a role in regulating structural genes needs further study. These findings can help to elucidate the molecular mechanism and regulatory networks of anthocyanin biosynthesis in walnut and provide a biological basis for breeding new walnut cultivars. 


\section{Conclusions}

In this study, metabolomics and transcriptomics were used to reveal the anthocyanin biosynthesis metabolic pathway. A total of 26 anthocyanins in SR were significantly upaccumulated comparing with $\mathrm{SG}$, and the transcriptome analysis demonstrated that the expression of structural genes $(\mathrm{C} 4 \mathrm{H}, \mathrm{F} 3 \mathrm{H}$, $F 3^{\prime} 5^{\prime} H$ and UFGTS), four MYBs, six WD40s in anthocyanin biosynthetic pathway were significantly higher in SR walnut. Delphinidin 3-0-galactoside, cyanidin 3-0-galactoside, delphinidin 3-0-glucoside and cyanidin 3-0-glucoside were identified as the primary components of anthocyanidins because of their higher contents. For proanthocyanin, proanthocyanin C1 was upregulated in SR compared with SG, while proanthocyanin B1 and proanthocyanin B3 were upregulated in SR-1 and SR-3 but downregulated in SR-2 compared with the controls. Our results provide valuable information on anthocyanin and PA metabolites and candidate genes in anthocyanin and PA biosynthesis, which provides new insights into anthocyanin and PA biosynthesis in walnuts.

\section{Methods}

\section{Plant material and growth conditions}

Red walnut (Juglans regia L. accession RW-1, germplasm resource number: JUREG4108210002) was introduced from Taihang Mountain, China (Fig. S1). To maintain a relatively consistent genetic background, various color phenotypes of natural hybrid plants (SG for green leaf natural hybrid plants and SR for red leaf natural hybrid plants) were grown in the Fruit Tree Experimental Station of Horticulture College, Henan Agricultural University, Zhengzhou, Henan, China. Sampling permission was obtained from the public land management agency of Henan Agricultural University. The leaves were collected according to the color change of SR walnut leaves. During the full red period (SR-1), red-green period (SR-2), and full green period (SR-3), the leaves of SG walnut from the same period of development were collected as the control [34] (Fig. 1a). All samples were immediately frozen in liquid nitrogen and stored at $-80^{\circ} \mathrm{C}$ until RNA and metabolite extraction.

\section{Extraction and quantification of metabolites}

The sample widely targeted metabolome and anthocyanidin detection were performed at Wuhan MetWare Biotechnology Co., Ltd. (Wuhan, China) as previously described [38]. In brief, $100 \mathrm{mg}$ leaves were crushed into powder and extracted with $0.6 \mathrm{~mL}$ of $70 \%$ aqueous methanol overnight at $4^{\circ} \mathrm{C}$ [49]. Following centrifugation at $10,000 \mathrm{~g}$ for $10 \mathrm{~min}$, the supernatant was filtered through a microporous membrane $(0.22 \mu \mathrm{m})$ for subsequent liquid chromatography/tandem mass spectrometry (LC-MS/MS) analysis.

Principal component analysis (PCA) was performed to verify the differences and reliability of metabolites in all the samples. Differentially accumulated metabolites (DAMs) between groups were filtered with VIP value $\geq 1$ and absolute $\log _{2} \mathrm{FC}$ (fold change) $\geq 1$. Subsequently, the differentially expressed metabolites with significant enrichment were mapped to the Kyoto Encyclopedia of Genes and Genomes (KEGG, 
https://www.genome.jp/kegg), and a plant metabolic network (PMN, https://plantcyc.org/) was used to analyze the phenylalanine metabolism pathway, flavonoid metabolism pathway and anthocyanin biosynthesis pathway $[49,50]$. We constructed metabolic pathways based on the KEGG database.

\section{RNA extraction, sequencing and transcriptome data analysis}

Total RNA from walnut leaves was extracted using the Omega Plant RNA Kit (Omega Biotek, Norcross, Georgia) according to the manufacturer's instructions. The integrity and quality of total RNA were examined on $1 \%$ agarose gels, and the RNA concentrations were measured by a NanoDrop 1000 spectrophotometer (Thermo Fisher Scientific, Waltham, MA, United States). A cDNA library was constructed and sequenced on a HiSeq 2,500 platform (Illumina, San Diego, CA, United States) (NCBI accession PRJNA688391) by Biomarker Biotechnology Corp. (Beijing, China), following paired-end reads were produced after cluster generation.

The expression abundance of unigenes was represented as FPKM (fragments per kilobase of transcript per million fragments mapped) values, and the DEGs [false discovery rate $(\mathrm{FDR})<0.05$, and $\left|\log _{2} \mathrm{FC}\right| \geq$ 1.5] between red leaves and green leaves were obtained using the DESeq 1.8.3 package [51]. Functional annotation was performed using Gene Ontology (GO), and the pathways with significant enrichment were identified based on KEGG pathways employing the cluster Profiler $R$ package [52].

\section{Quantitative Real-time (qRT) PCR Assay}

The expression of structural genes and transcription factor genes in the anthocyanin biosynthetic pathway was examined by qRT-PCR. First-stand CDNA was synthesized using the FastQuant RT Kit (with gDNase) (Tiangen Biotech, Beijing, China), and qRT-PCR was performed using ChamQ Universal SYBR qPCR Master Mix (Vazyme, Nanjing, China) on an ABI 7500 Real-Time PCR system (Applied Biosystems, Foster City, CA, United States). Jr18S (GenBank accession No. XM_019004991.1) was used as the housekeeping gene [53]. Quantification was evaluated using the $2-\Delta \Delta \mathrm{Ct}$ method. All the primers are shown in Table S1.

\section{Statistical analysis}

All data were analyzed using SPSS 22.0 software, expressed as the mean \pm standard deviation (SD) of three replicates. Significant differences were carried out using a one-sided paired t-test $\left({ }^{\star *} p<0.01,{ }^{*} p<\right.$ 0.05 ) between red leaf and green leaf samples.

\section{Abbreviations}

ANR: anthocyanidin reductase

ANS: anthocyanin synthetase

C4H: cinnamate 4-hydroxylase 
$\mathrm{CHI} \bowtie$ chalcone isoenzyme

$\mathrm{CHS} \llbracket$ chalcone synthetase

Cy『Cyanidin

DAMs: differentially accumulated metabolites

DEGs: the different expressed genes

DFR: dihydroflavonol 4-reductase

Dp囚delphinidin

F3H: flavanone 3-hydroxylase

F3'5'H: flavonoid 3',5'-Hydroxylase

FDR: false discovery rate

FPKM: fragments per kilobase of transcript per million fragments mapped

GO: Gene Ontology

KEGG: Kyoto Encyclopedia of Genes and Genomes

LAR: leucoanthocyanidin reductase

LC-MS/MS: liquid chromatography/tandem mass spectrometry

Mv囚malvidin

PA: proanthocyanin

PAL囚phenylalanine ammonia lyase

PCA: principal component analysis

Pg: pelargonidin

Pn囚peonidin

Pt\petunidin

QC: quality control

RW-1هred walnut 'RW-1' 
SG: the green-leaf walnuts of RW-1 natural hybrid progenies

SR: the red-leaf walnuts of RW-1 natural hybrid progenies

UFGT囚UDP glucose: flavonoid-3-0-glucosyltransferase

UPLC-ESI-MS/MS: ultra-performance liquid chromatography coupled to electrospray ionization tandem mass spectrometry

\section{Declarations}

Ethics approval and consent to participate

The collection of wild red walnut was completed under the support of the project 'Investigation and cataloging of walnut genetic resources in Henan Province (2016) (Grant ID:GR-2016-06)', and all the experimental research and field studies on plants comply with the IUCN Policy Statement on Research Involving Species at Risk of Extinction and the Convention on the Trade in Endangered Species of Wild Fauna and Flora.

Consent for publication

Not applicable.

Availability of data and materials

The datasets presented in this study can be found in online repositories. The name of the repository and accession numbers can be found at the National Center for Biotechnology Information, https://www.ncbi.nlm.nih.gov/, PRJNA688391.

Competing interests

The authors declare that they have no competing interests.

Funding

This work was supported by the National Key R\&D Program of China (2018YFD1000604) and the Support Plan of Scientific and Technological Innovation Team in Colleges and Universities of Henan Province, China (19IRTSTHN009). The funding organizations provided financial support to the research projects but had no role in the design of the study, data collection and analysis, or writing of the manuscript.

Authors' Contributions

GW and JS designed the research. LL and WZ performed experimental work and data analysis and prepared the original draft. LL and LW participated in data analysis; $H M, G Z$ and WW helped review and 
edit the draft; LW provided support for projects and funds and revised the manuscript. All authors have read and agreed to the published version of the manuscript.

Acknowledgments

Not applicable.

\section{References}

1. Cho K, Cho K-S, Sohn H-B, Ha IJ, Hong S-Y, Lee H, Kim Y-M, Nam MH: Network analysis of the metabolome and transcriptome reveals novel regulation of potato pigmentation. Journal of experimental botany 2016, 67(5):1519-1533.

2. Honda C, Moriya S: Anthocyanin biosynthesis in apple fruit. The Horticulture Journal 2018, 87(3):305-314.

3. Sasaki N, Nishizaki Y, Ozeki Y, Miyahara T: The role of acyl-glucose in anthocyanin modifications. Molecules 2014, 19(11):18747-18766.

4. Shi M-Z, Xie D-Y: Biosynthesis and metabolic engineering of anthocyanins in Arabidopsis thaliana. Recent patents on biotechnology 2014, 8(1):47-60.

5. Marles MS, Ray H, Gruber MY: New perspectives on proanthocyanin biochemistry and molecular regulation. Phytochemistry 2003, 64(2):367-383.

6. Dixon RA, Xie DY, Sharma SB: proanthocyanins-a final frontier in flavonoid research? New phytologist 2005, 165(1):9-28.

7. Li H, Yang Z, Zeng Q, Wang S, Luo Y, Huang Y, Xin Y, He N: Abnormal expression of bHLH3 disrupts a flavonoid homeostasis network, causing differences in pigment composition among mulberry fruits. Horticulture research 2020, 7(1):1-19.

8. Jun JH, Xiao X, Rao X, Dixon RA: proanthocyanin subunit composition determined by functionally diverged dioxygenases. Nature plants 2018, 4(12):1034-1043.

9. Saigo $T$, Wang $T$, Watanabe $M$, Tohge $T$ : Diversity of anthocyanin and proanthocyanin biosynthesis in land plants. Current opinion in plant biology 2020, 55:93-99.

10. Katsumoto Y, Fukuchi-Mizutani M, Fukui Y, Brugliera F, Holton TA, Karan M, Nakamura N, YonekuraSakakibara K, Togami J, Pigeaire A: Engineering of the rose flavonoid biosynthetic pathway successfully generated blue-hued flowers accumulating delphinidin. Plant and Cell Physiology 2007, 48(11):15891600 . 
11. Caputi L, Malnoy M, Goremykin V, Nikiforova S, Martens S: A genome-wide phylogenetic reconstruction of family 1 UDP-glycosyltransferases revealed the expansion of the family during the adaptation of plants to life on land. The Plant Journal 2012, 69(6):1030-1042.

12. Yonekura-Sakakibara K, Fukushima A, Nakabayashi R, Hanada K, Matsuda F, Sugawara S, Inoue E, Kuromori T, Ito T, Shinozaki $\mathrm{K}$ et al: Two glycosyltransferases involved in anthocyanin modification delineated by transcriptome independent component analysis in Arabidopsis thaliana. The Plant Journal 2012, 69(1):154-167.

13. Zhang Z, Tian C, Zhang Y, Li C, Li X, Yu Q, Wang S, Wang X, Chen X, Feng S: Transcriptomic and metabolomic analysis provides insights into anthocyanin and procyanidin accumulation in pear. $B M C$ plant biology 2020, 20:1-14.

14. Matus JT, Cavallini E, Loyola R, Höll J, Finezzo L, Dal Santo S, Vialet S, Commisso M, Roman F, Schubert A: A group of grapevine MYBA transcription factors located in chromosome 14 control anthocyanin synthesis in vegetative organs with different specificities compared with the berry color locus. The Plant Journal 2017, 91(2):220-236.

15. Vimolmangkang S, Han Y, Wei G, Korban SS: An apple MYB transcription factor, MdMYB3, is involved in regulation of anthocyanin biosynthesis and flower development. BMC plant biology 2013, 13(1):1-13.

16. Zhong C, Tang Y, Pang B, Li X, Yang Y, Deng J, Feng C, Li L, Ren G, Wang Y: The R2R3-MYB transcription factor GhMYB1a regulates flavonol and anthocyanin accumulation in Gerbera hybrida. Horticulture Research 2020, 7(1):1-13.

17. Nesi N, Jond C, Debeaujon I, Caboche M, Lepiniec L: The Arabidopsis TT2 gene encodes an R2R3 MYB domain protein that acts as a key determinant for proanthocyanin accumulation in developing seed. The Plant Cell 2001, 13(9):2099-2114.

18. Bogs J, Jaffé FW, Takos AM, Walker AR, Robinson SP: The grapevine transcription factor VvMYBPA1 regulates proanthocyanin synthesis during fruit development. Plant physiology 2007, 143(3):1347-1361.

19. Terrier N, Torregrosa L, Ageorges A, Vialet S, Verries C, Cheynier V, Romieu C: Ectopic expression of VvMybPA2 promotes proanthocyanin biosynthesis in grapevine and suggests additional targets in the pathway. Plant physiology 2009, 149(2):1028-1041.

20. Bai YC, Li CL, Zhang JW, Li SJ, Luo XP, Yao HP, Chen H, Zhao HX, Park SU, Wu Q: Characterization of two tartary buckwheat R2R3-MYB transcription factors and their regulation of proanthocyanin biosynthesis. Physiologia Plantarum 2014, 152(3):431-440. 
21. Liu C, Jun JH, Dixon RA: MYB5 and MYB14 play pivotal roles in seed coat polymer biosynthesis in Medicago truncatula. Plant physiology 2014, 165(4):1424-1439.

22. Lu N, Roldan M, Dixon RA: Characterization of two TT2-type MYB transcription factors regulating proanthocyanin biosynthesis in tetraploid cotton, Gossypium hirsutum. Planta 2017, 246(2):323-335.

23. Hichri I, Barrieu F, Bogs J, Kappel C, Delrot S, Lauvergeat V: Recent advances in the transcriptional regulation of the flavonoid biosynthetic pathway. Journal of experimental botany 2011, 62(8):2465-2483.

24. Springob K, Nakajima J-i, Yamazaki M, Saito K: Recent advances in the biosynthesis and accumulation of anthocyanins. Natural product reports 2003, 20(3):288-303.

25. Wang L, Tang W, Hu Y, Zhang Y, Sun J, Guo X, Lu H, Yang Y, Fang C, Niu X: A MYB/bHLH complex regulates tissue-specific anthocyanin biosynthesis in the inner pericarp of red-centered kiwifruit Actinidia chinensis cv. Hongyang. The Plant Journal 2019, 99(2):359-378.

26. Li C, Wu J, Hu K-D, Wei S-W, Sun H-Y, Hu L-Y, Han Z, Yao G-F, Zhang H: PyWRKY26 and PybHLH3 cotargeted the PyMYB114 promoter to regulate anthocyanin biosynthesis and transport in red-skinned pears. Horticulture research 2020, 7(1):1-12.

27. Baudry A, Heim MA, Dubreucq B, Caboche M, Weisshaar B, Lepiniec L: TT2, TT8, and TTG1 synergistically specify the expression of BANYULS and proanthocyanin biosynthesis in Arabidopsis thaliana. The Plant Journal 2004, 39(3):366-380.

28. Schaart JG, Dubos C, Romero De La Fuente I, van Houwelingen AM, de Vos RC, Jonker HH, Xu W, Routaboul JM, Lepiniec L, Bovy AG: Identification and characterization of MYB-b HLH-WD 40 regulatory complexes controlling proanthocyanin biosynthesis in strawberry ( $\mathrm{F}$ ragariax ananassa) fruits. New phytologist 2013, 197(2):454-467.

29. del Mar Naval M, Gil-Muñoz F, Lloret A, Besada C, Salvador A, Badenes ML, Ríos G: A WD40-repeat protein from persimmon interacts with the regulators of proanthocyanin biosynthesis DkMYB2 and DkMYB4. Tree Genetics \& Genomes 2016, 12(1):13.

30. Xu W, Dubos C, Lepiniec L: Transcriptional control of flavonoid biosynthesis by MYB-bHLH-WDR complexes. Trends in plant science 2015, 20(3):176-185.

31. Li Y, Luo X, Wu C, Cao S, Zhou Y, Jie B, Cao Y, Meng H, Wu G: Comparative transcriptome analysis of genes involved in anthocyanin biosynthesis in red and green walnut (Juglans regia L.). Molecules 2018, 23(1):25.

32. Yang; $Y$, Wang; L, Liu; $Y, W u ; W$, Meng; $H$, Zhao; W, Li; L, Wu G: Comparison of leaf traits and diumal variation of photosynthetic rates between red- and green-leafed walnuts. Journal of Fruit Science 2020, 37(8):1175-1183. 
33. Zhao W, Liu Y, Li L, Meng H, Yang Y, Dong Z, Wang L, Wu G: Genome-wide identification and characterization of bHLH transcription factors related to anthocyanin biosynthesis in red walnut (Juglans regia L.). Frontiers in genetics 2021, 12.

34. Zhao W, Li L, Liu Y, Zhang L, Yang Y, Meng H, Wang L, Wu G: Identification and bioinformatics analysis of CHS genes in different phenotypic leaves of natural hybrid progenies of red-kernel walnut. Journal of Fruit Science 2021, 38(02):179-191.

35. Shi Q, Du J, Zhu D, Li X, Li X: Metabolomic and Transcriptomic Analyses of Anthocyanin Biosynthesis Mechanisms in the Color Mutant Ziziphus jujuba cv. Tailihong. Journal of Agricultural and Food Chemistry 2020, 68(51):15186-15198.

36. Solhaug EM, Roy R, Chatt EC, Klinkenberg PM, Mohd-Fadzil NA, Hampton M, Nikolau BJ, Carter CJ: An integrated transcriptomics and metabolomics analysis of the Cucurbita pepo nectary implicates key modules of primary metabolism involved in nectar synthesis and secretion. Plant direct 2019, 3(2): 000120 .

37. Yang B, He S, Liu Y, Liu B, Ju Y, Kang D, Sun X, Fang Y: Transcriptomics integrated with metabolomics reveals the effect of regulated deficit irrigation on anthocyanin biosynthesis in Cabernet Sauvignon grape berries. Food chemistry 2020, 314:126170.

38. Zhang Q, Wang L, Liu Z, Zhao Z, Zhao J, Wang Z, Zhou G, Liu P, Liu M: Transcriptome and metabolome profiling unveil the mechanisms of Ziziphus jujuba Mill. peel coloration. Food chemistry 2020, 312:125903.

39. Zhuang H, Lou Q, Liu H, Han H, Wang Q, Tang Z, Ma Y, Wang H: Differential regulation of anthocyanins in green and purple turnips revealed by combined de novo transcriptome and metabolome analysis. International journal of molecular sciences 2019, 20(18):4387.

40. Li Y, Chen Q, Xie X, Cai Y, Li J, Feng Y, Zhang Y: Integrated Metabolomics and Transcriptomics Analyses Reveal the Molecular Mechanisms Underlying the Accumulation of Anthocyanins and Other Flavonoids in Cowpea Pod (Vigna unguiculata L.). Journal of Agricultural and Food Chemistry 2020, 68(34):9260-9275.

41. Petroni K, Tonelli C: Recent advances on the regulation of anthocyanin synthesis in reproductive organs. Plant science 2011, 181(3):219-229.

42. Naing AH, Kim CK: Roles of R2R3-MYB transcription factors in transcriptional regulation of anthocyanin biosynthesis in horticultural plants. Plant molecular biology 2018, 98(1):1-18.

43. Jiang T, Zhang M, Wen C, Xie X, Tian W, Wen S, Lu R, Liu L: Integrated metabolomic and transcriptomic analysis of the anthocyanin regulatory networks in Salvia miltiorrhiza Bge. flowers. $B M C$ Plant Biology 2020, 20(1):1-13. 
44. Tanaka Y, Sasaki N, Ohmiya A: Biosynthesis of plant pigments: anthocyanins, betalains and carotenoids. The Plant Journal 2008, 54(4):733-749.

45. Liu H, Su J, Zhu Y, Yao G, Allan AC, Ampomah-Dwamena C, Shu Q, Lin-Wang K, Zhang S, Wu J: The involvement of PybZIPa in light-induced anthocyanin accumulation via the activation of PyUFGT through binding to tandem G-boxes in its promoter. Horticulture research 2019, 6(1):1-13.

46. Koes R, Verweij W, Quattrocchio F: Flavonoids: a colorful model for the regulation and evolution of biochemical pathways. Trends in plant science 2005, 10(5):236-242.

47. Montefiori M, Espley RV, Stevenson D, Cooney J, Datson PM, Saiz A, Atkinson RG, Hellens RP, Allan AC: Identification and characterisation of F3GT1 and F3GGT1, two glycosyltransferases responsible for anthocyanin biosynthesis in red-fleshed kiwifruit (Actinidia chinensis). The Plant Journal 2011, 65(1):106-118.

48. Van Nocker S, Ludwig P: The WD-repeat protein superfamily in Arabidopsis: conservation and divergence in structure and function. BMC genomics 2003, 4(1):1-11.

49. Wang Z, Cui Y, Vainstein A, Chen S, Ma H: Regulation of fig (Ficus carica L.) fruit color: metabolomic and transcriptomic analyses of the flavonoid biosynthetic pathway. Frontiers in Plant Science 2017, 8:1990.

50. Viant MR, Kurland IJ, Jones MR, Dunn WB: How close are we to complete annotation of metabolomes? Current opinion in chemical biology 2017, 36:64-69.

51. Yi D, Zhang H, Lai B, Liu L, Pan X, Ma Z, Wang Y, Xie J, Shi S, Wei Y: Integrative Analysis of the Coloring Mechanism of Red Longan Pericarp through Metabolome and Transcriptome Analyses. Journal of Agricultural and Food Chemistry 2020.

52. Yu G, Wang L-G, Han Y, He Q-Y: clusterProfiler: an R package for comparing biological themes among gene clusters. Omics: a journal of integrative biology 2012, 16(5):284-287.

53. Speer H, D'Cunha NM, Alexopoulos NI, McKune AJ, Naumovski N: Anthocyanins and human healthA focus on oxidative stress, inflammation and disease. Antioxidants 2020, 9(5):366.

\section{Figures}


a

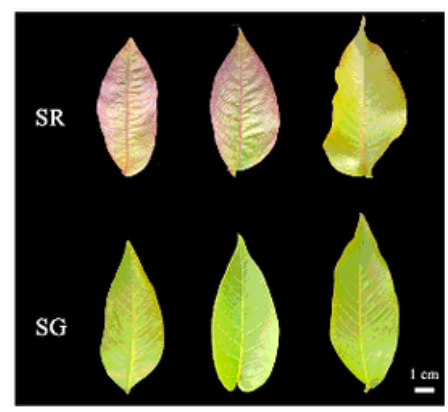

b

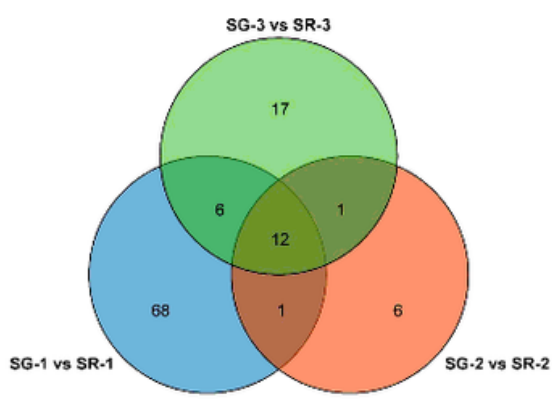

d
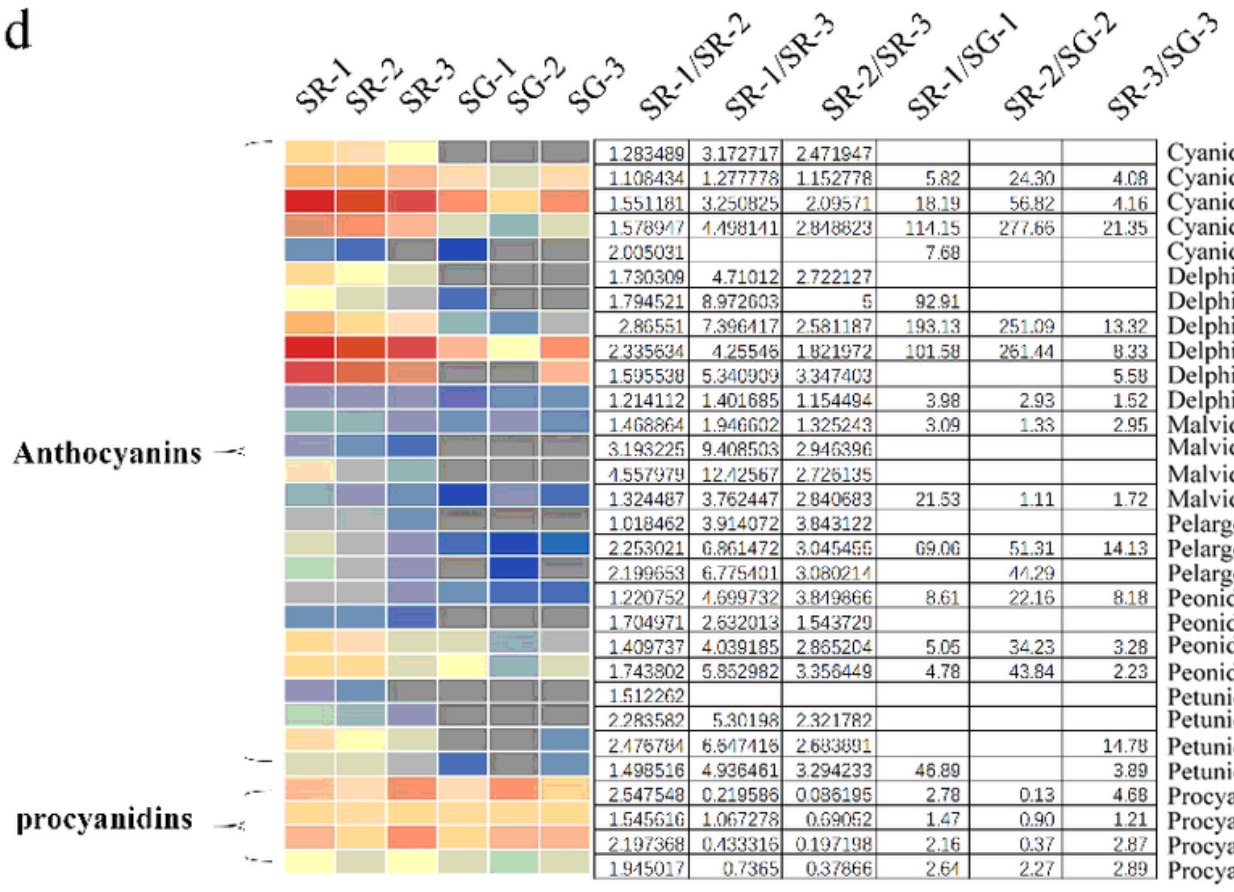

$\mathrm{C}$

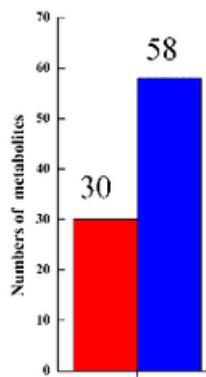
Down accumulated metabolites

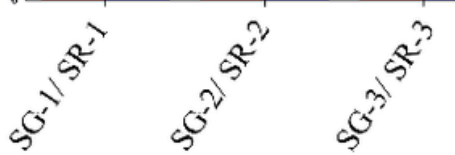

22

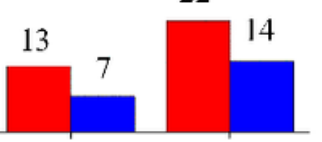

Cyanidin 3-O-(6-O-malonyl-beta-D-glucoside)

Cyanidin 3-O-arabinoside

Cyanidin 3-O-galactoside

Cyanidin 3-O-glucoside

Cyanidin 3-O-rutinoside

Delphinidin 3,5-O-diglucoside(Delphin)

Delphinidin 3-O-(6"-O-malonyl)-beta-D-glucoside

Delphinidin 3-O-arabinoside

Delphinidin 3-O-galactoside

Delphinidin 3-O-glucoside

Delphinidin 3-O-rutinoside

Malvidin 3-O-(6-O-malonyl-beta-D-glucoside)

Malvidin 3-O-arabinoside

Malvidin 3-O-galactoside

Malvidin 3-O-glucoside

Pelargonidin 3-O-(6-O-malonyl-beta-D-glucoside)

Pelargonidin 3-O-arabinoside

Pelargonidin 3-O-glucoside

Peonidin 3-O-glucoside

Peonidin 3-O-(6-O-malonyl-beta-D-glucoside)

Peonidin 3-O-galactoside

Peonidin 3-O-galactoside

Petunidin 3-O-(6-O-malonyl-beta-D-glucoside)

Petunidin 3-O-arabinoside

Petunidin 3-O-galactoside

Petunidin 3-O-glucoside

Procyanidin B1

Procyanidin $\mathrm{B} 2$

Procyanidin B2

Procyanidin $\mathrm{B} 3$
Procyanidin $\mathrm{Cl}$

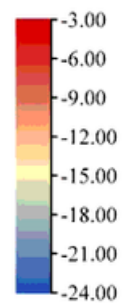

\section{Figure 1}

Metabolite profiles between SG and SR. a Morphological observation of leaves of different colors in natural hybrid progenies of red walnut. b Venn diagram depicting the shared and specific metabolites between the three compared groups of leaf samples. c Number of differentially accumulated metabolites (DAMs) in SG-1 vs. SR-1, SG-2 vs. SR-2 and SG-3 vs. SR-3. d Concentrations of anthocyanins in leaves of SG and SR. Numbers refer to -fold change in anthocyanin contents. 


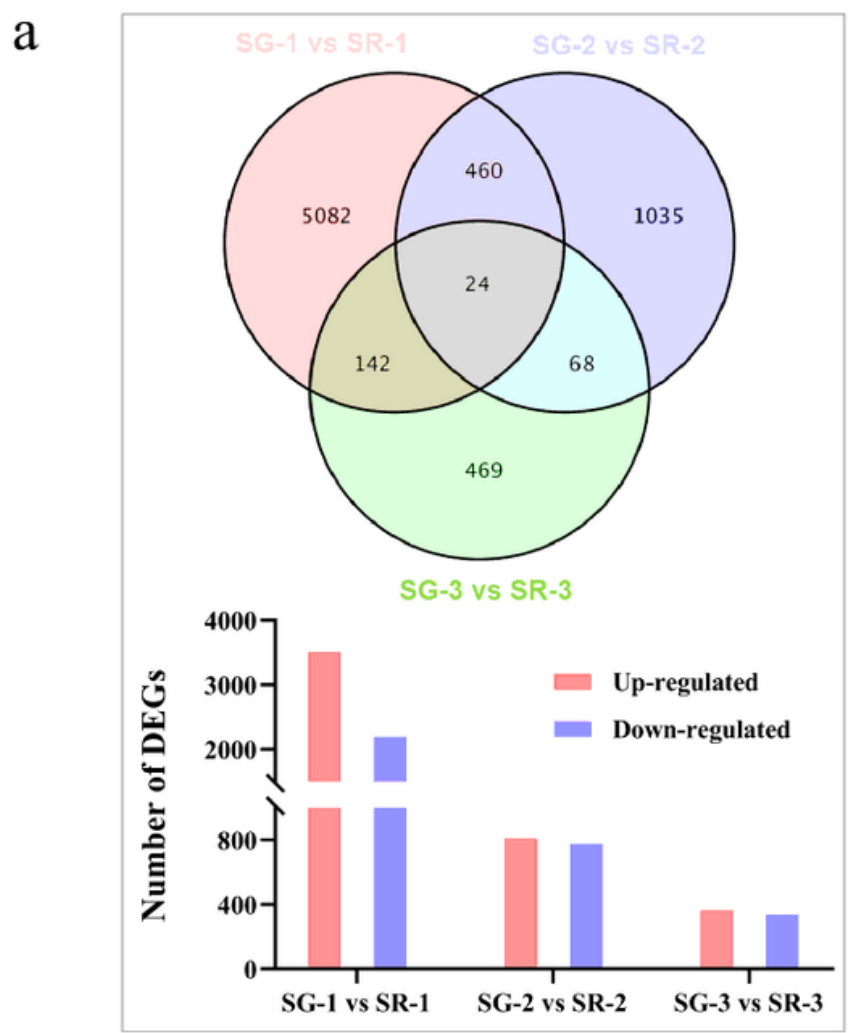

b

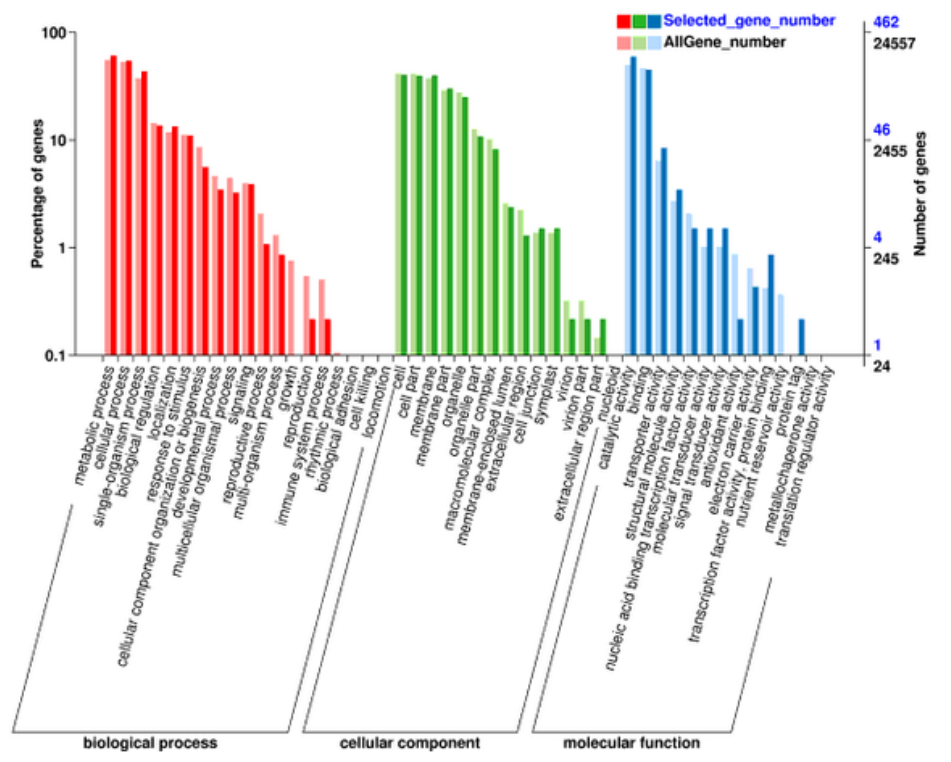

C

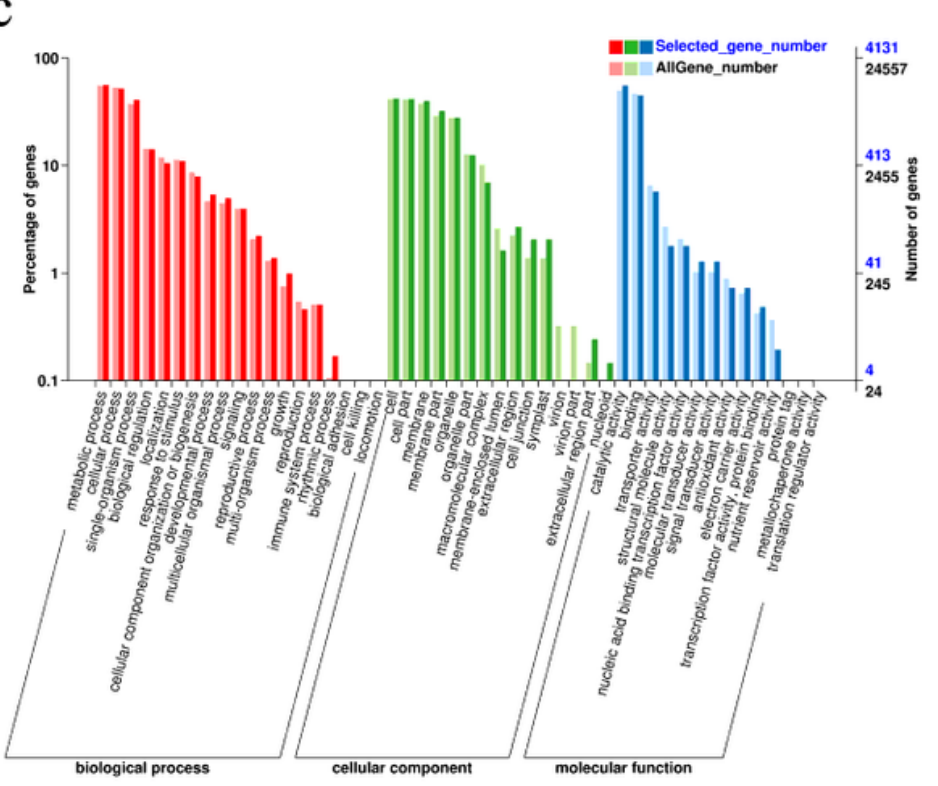

d

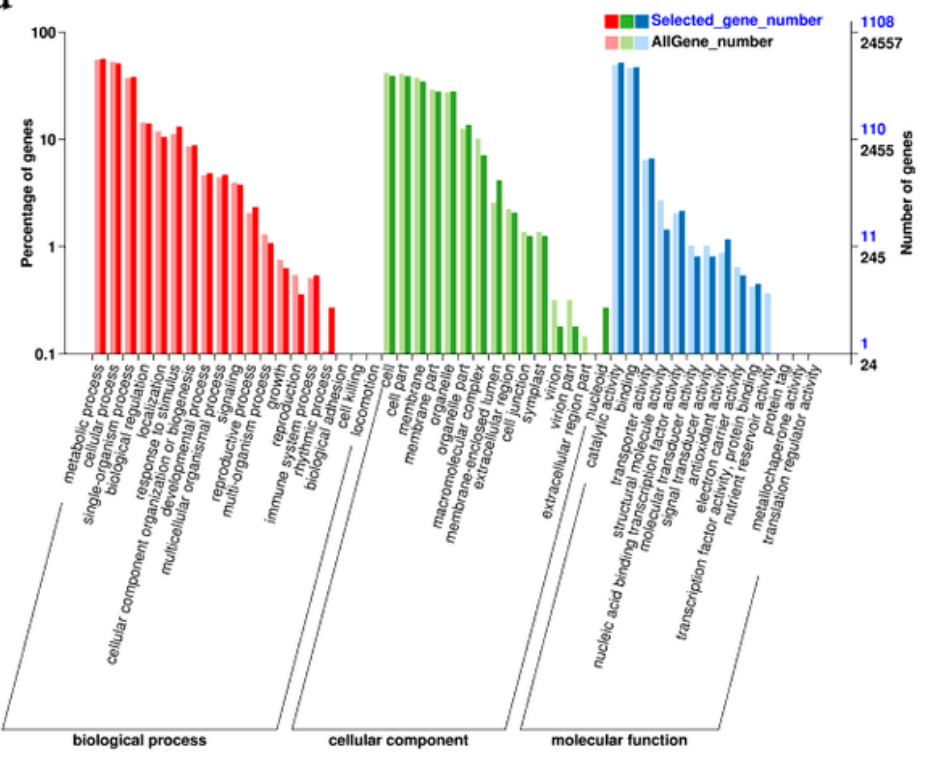

Figure 2

Number and GO enrichment profiles of differentially expressed genes (DEGs) identified by RNA-seq analysis. a Venn diagram and histogram representing the numbers of DEGs. $\mathbf{b}-\mathbf{d}$ GO annotations of DEGs in SG-1 vs. SR-1 (b), SG-2 vs. SR-2 (c) and SG-3 vs. SR-3 (d). 


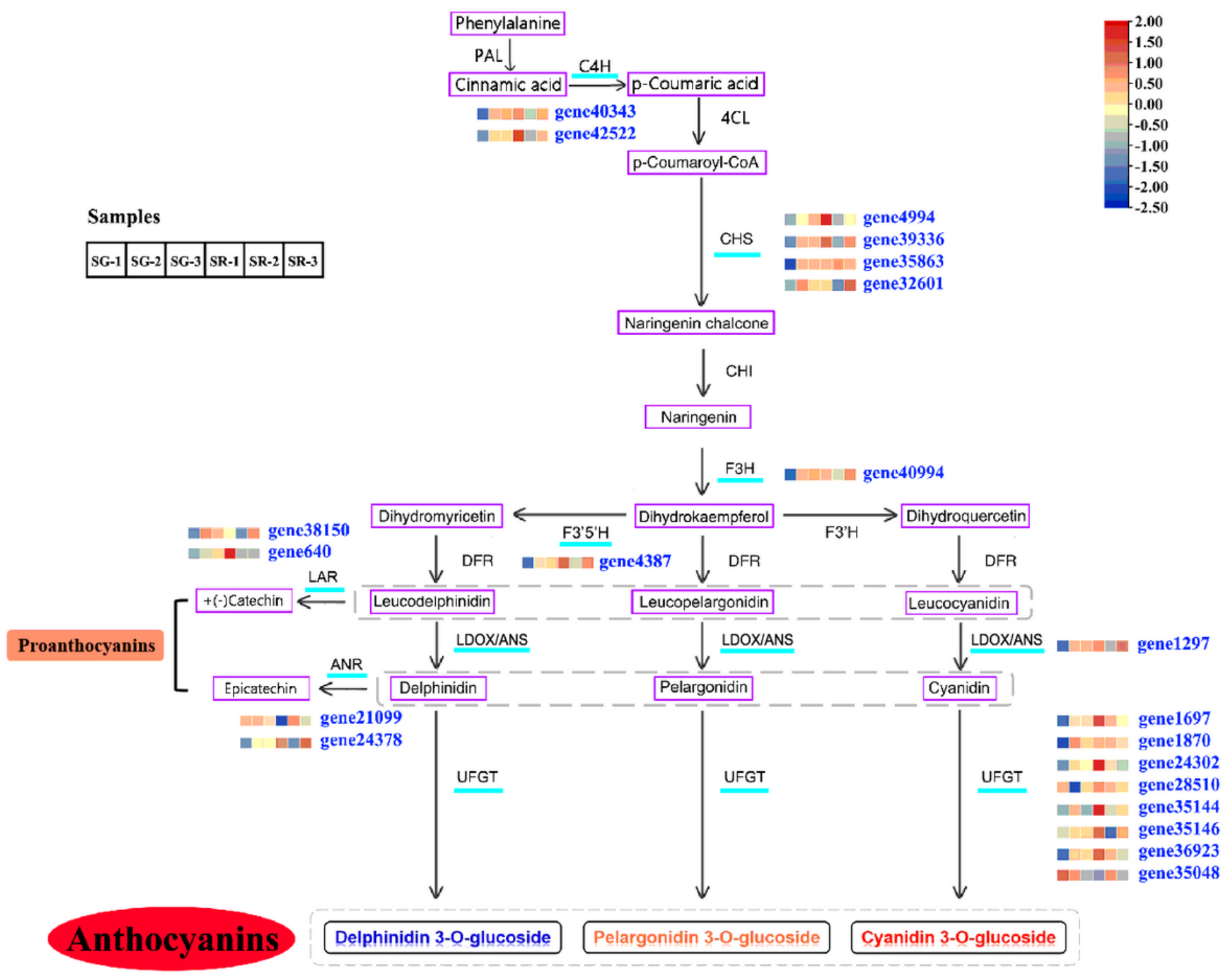

\section{Figure 3}

Modulation of anthocyanin and PA biosynthesis pathway genes during walnut leaf development. The heatmap represents the expression of corresponding structural genes in SR and SG, and blue to red in the heatmap indicates the expression levels of structural genes (FPKM value) ranging from low to high. PAL, phenylalanine ammonia lyase; $\mathrm{C} 4 \mathrm{H}$, cinnamate 4-hydroxylase; $4 \mathrm{CL}$, 4-coumarate: CoA ligase; $\mathrm{CHS}$, chalcone synthase; $\mathrm{CHI}$, chalcone isomerase; $\mathrm{F3H}$, flavanone 3-hydroxylase; $\mathrm{F3}$ 'H, flavonoid 3'hydroxylase; F3'5'H, flavonoid 3',5'-hydroxylase; DFR, dihydroflavonol 4-reductase; ANS, anthocyanidin synthase; UFGT, UDP-glucose: flavonoid 3-Oglucosyltransferase; LAR, leucoanthocyanidin reductase; ANR, anthocyanidin reductase. 
a

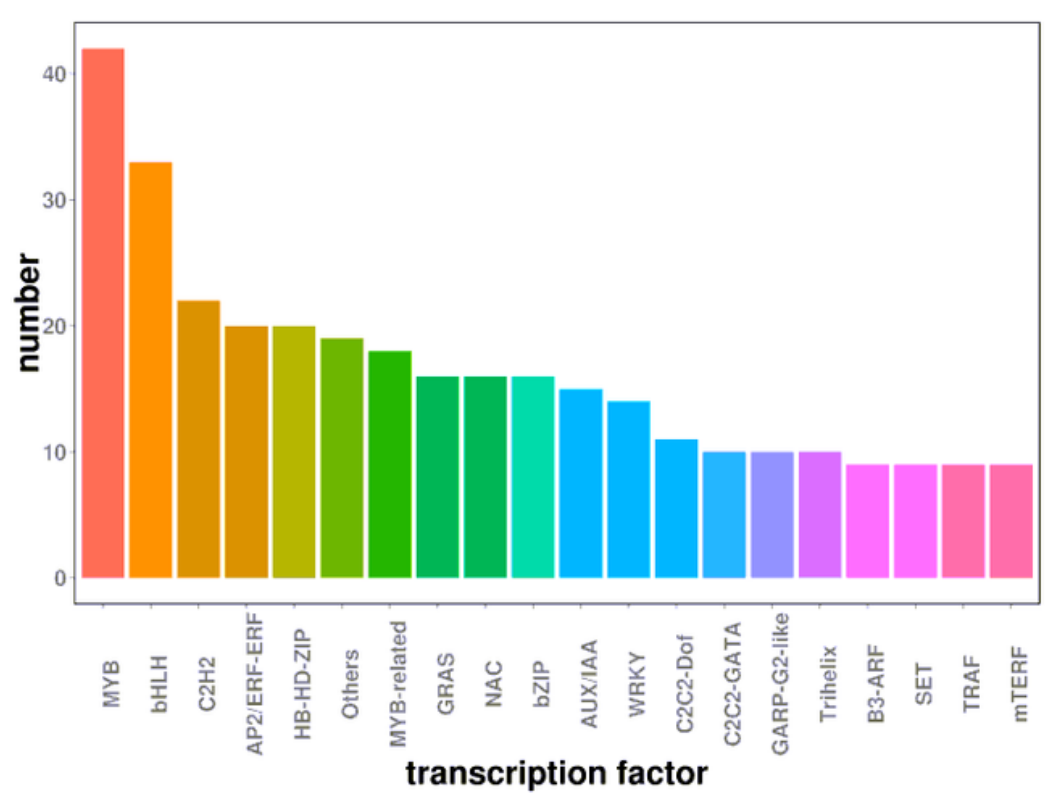

$\mathrm{b}$

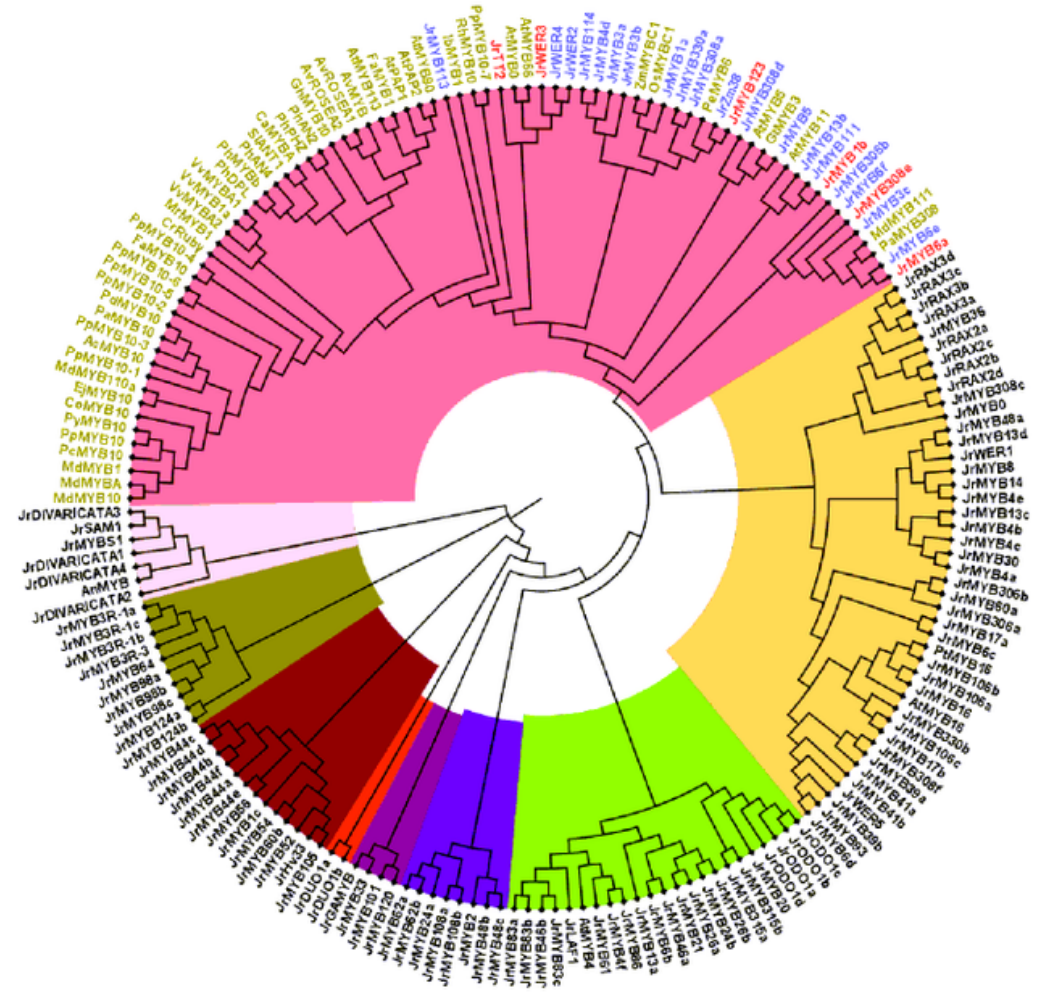

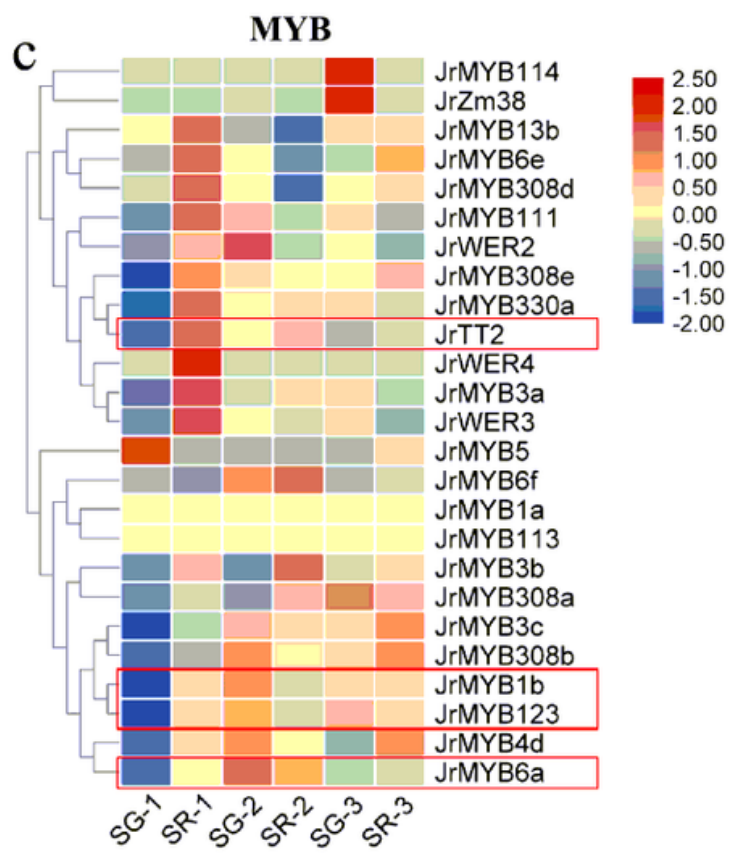

d

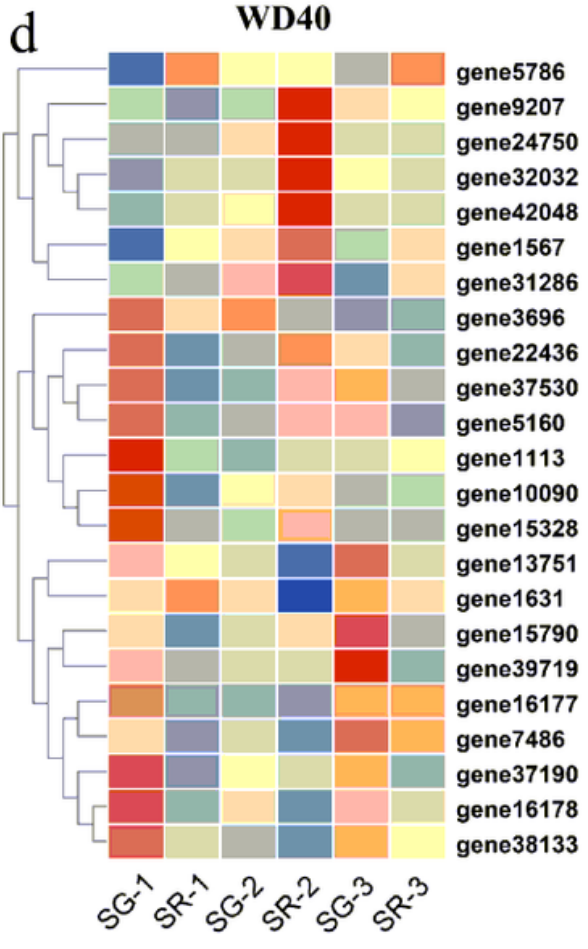

Figure 4

Analysis of transcription factors related to anthocyanin biosynthesis. a Number of differentially expressed transcription factors. b Phylogenetic tree of MYB proteins between walnut, Arabidopsis thaliana, and MYBs related to anthocyanin biosynthesis in other species. c Expression heatmap of MYB highly homologous to anthocyanin-related MYBs of other species. $\mathbf{d}$ Expression heatmap of differentially expressed WD40 in SG and SR.

\section{Supplementary Files}


This is a list of supplementary files associated with this preprint. Click to download.

- SupplementalFiguresS1S6.docx

- SupplementalTablesS1S4.docx 